\title{
Three-Dimensional Modeling in Congenital and Structural Heart Perioperative Care and Education: A Path in Evolution
}

\author{
George E. Sarris $^{1}$ - Anastasios C. Polimenakos ${ }^{2}$
}

Received: 3 April 2017/ Accepted: 4 April 2017/Published online: 29 April 2017

(C) Springer Science+Business Media New York 2017

Three-dimensional (3D) printing is an emerging technology in the medical field, and, especially, in congenital and structural heart disease, its role has, yet, to be defined. Congenital heart surgeons and structural heart interventionalists are constantly seeking valuable tools to better understand complex anatomy, determine suitability of a corrective strategy, and develop a roadmap towards a customized operative or interventional approach $[1,2]$.

Intuitively, cardiovascular 3D printing seems to offer a powerful means for congenital cardiac surgeons, acquired heart disease surgeons, and interventional cardiologists to create patient-specific anatomy models and orchestrate an optimal therapeutic strategy, individualized to the patient. However, this technology has, yet, to attract routine and widespread application [3, 4]. This is likely driven in part by the technical limitations of bedside imaging (including 3D echocardiography) as well as those of advanced imaging (CT or MRI) to provide high-resolution data, the limitations of segmentation software to distinguish between tissues of very similar "image density," by current limitations in printing technology with materials that can be manipulated (cut, dissected, retracted, sutured) in ways meaningful to a surgeon, and, last but not least, the relatively high cost of production of the 3D models.

Anastasios C. Polimenakos

apolimenakos@augusta.edu

1 Division of Pediatric Cardiothoracic Surgery, Athens Heart Surgery Institute, Iaso Children's Hospital, Athens, Greece

2 Division of Pediatric Cardiothoracic Surgery, Children's Hospital of Georgia Heart Center, Medical College of Georgia, 1120 15th Street BAA 8300, Augusta, GA 30912, USA
Potential applications of 3D printing in Congenital and Structural Heart Surgery and Interventions are in evolution. To date, cardiovascular 3D prints have been mainly used as a complimentary adjunct to the imaging modalities from which they are derived $[5,6]$. The potential benefits of a "static" physical model are predominantly related to an enhanced spatial understanding of a structurally complex native, post-surgical or post-interventional cardiovascular malformation. Such hands-on understanding might, then, facilitate formation of a technically sound operative or interventional approach.

As technology evolves, sophisticated care practice models, customized to the individual patient, and operative or interventional simulations in education are advocated.

Concepts, where 3D cardiovascular printing might gain widespread acceptance, can be directed towards:

A. Potential benefits to the individual patient from:

(1) Family consultation with stepwise basic education of patient's specific anatomical characteristics This provides not only visual engagement of the families with the verbalized description of heart morphology, but also allows the physician to emphasize the associated risks and specific steps of the procedure that might prognosticate a favorable or non-favorable outcome.

(2) Rehearsal and simulation in alternative preoperative scenarios by applying surgical strategies or variations Combined with the growing experience and data regarding surgical simulation, 3D printing is poised to enable widespread patient-specific deliberate practice, akin to flight simulation in the aviation industry. Operative rehearsal with $3 \mathrm{D}$ prints may be meaningful to surgeons at all levels of skill and experience, 
depending on the specific circumstance. As a recent study [8] highlighted, these type of rehearsals with patient-specific 3D prints may reveal to a surgeon potential pitfalls, a preferred approach, or optimal instrumentation, at the same time providing a preview of anatomic nuance and patient-specific deliberate practice of a planned operation. The authors established a model (hydrogel medium print) on 1:1 scale which allowed realistic creation of the operative resection experience. In both cases, a dominant specimen was resected first and multiple smaller pieces were resected subsequently, just as is usual practice in the operating room.

(3) Enhancement of ICU conceptualization for the specific morphology of the anatomical lesion This will allow 3D or 4D virtual simulation of potential postoperative complications associated with the lesion-specific operative model and facilitate management within the workframe of a multidisciplinary non-operative team [9]. Conventional handoff represents the cornerstone for transferring clinical information across disciplines. Focusing on a, relatively exclusive, verbal transfer of critical information, conventional handoff highlights key clinically specialized data and fixed expectations as delivered by health providers with enhanced understanding of the anatomical nuances and surgical strategies. The addition of $3 \mathrm{D}$ or $4 \mathrm{D}$ virtual simulation conveys a refined assimilation of broader operative or interventional understanding, streamlines management strategies in clinical scenarios altered based on potential complications that might occur during the patient's postoperative course, and facilitates the quality of perception across different disciplines irrespective of health provider's anatomical or surgical background. As recently reported data suggested [9, 10], this can be particularly relevant when case-specific structural complexity is higher.

(4) Superior correlation with any given pathology and advanced delineation of anatomical details which can augment the step-by-step understanding of an individual case by the surgeon/ interventionalist and the entire perioperative team.

B. Potential role in education:

(1) Interactive education for tutoring students, trainees, and health care professionals This would allow not only a platform for basic and advanced anatomy and pathophysiology teaching, but also a venue where specific variations of a congenital anomaly or structural variation of anatomical configuration can be highlighted and conceptualized on an interactive constructive fashion.

(2) Training for the structural and congenital heart fellows in congenital heart surgery programs involving cases that advanced preoperative planning is mandated and those that, due to their scarcity, simulation is needed to visually narrate the anatomy and indicated corrective or palliative intervention. This can be particularly attractive in centers of low-volume where highrisk operations cannot serve as a platform for teaching or skill acquisition by the trainee. The ability for residents or fellows to learn and practice on 3D prints representing "real anatomy" and to compare resections done by attending and resident surgeons on identical $3 \mathrm{D}$ prints may enable a resident to demonstrate sufficient knowledge, skill, and confidence to learn and perform these cases while in training. This could be facilitated by the incorporation of specific anatomical modules into the Cardiac Surgery Simulation Curriculum [7].

(3) Creation of an ever expanding 3D cardiac model digital library of cardiovascular malformations from which cardiac models can be downloaded by users and printed for educational uses. Such libraries will initially supplement the existing few and precious true anatomical libraries, which are not, however, readily accessible. In the future, model libraries may supplant anatomical libraries for many of their functions.

It is beyond any doubt that this technology is here to stay. At present, although the potential benefits described above, related to enhancing preoperative health care team understanding, augmenting family education, and optimizing planning for and conduct of intraoperative and perioperative care (thereby increasing safety and effectiveness), are all intuitively profound, objective metrics to evaluate the impact of this technology on clinical outcomes are lacking.

There are no prospective randomized comparisons of the usefulness of cardiovascular 3D printing with conventional imaging strategies. There are no data pairing effective utilization and cost-effectiveness of this technology with improved clinical outcomes or educational objectives, thereby providing solid support for its widespread implementation. Currently, the European Congenital Heart Surgeons Association has launched a prospective registry study collecting data from participating European and 
North American Centers which utilize cardiac 3D Printing, with aiming to document the extent to which this technology is providing user assessed benefits in clinical practice, to define the types of cases where utility is most evident, and to provide information on the types of educational applications and their benefits. This study could provide the basis for more focused randomized studies.

Funding The author(s) received no financial support for the research, authorship, and/or publication of this article.

\section{Compliance with Ethical Standards}

Conflict of interest The author(s) declared no potential conflicts of interest with respect to the research, authorship, and/or publication of the article.

Informed consent The study was approved by the Institutional Review Board. Need for parental consent was waived.

\section{References}

1. Schmauss D, Schmitz C, Bigdeli AK, Weber S (2012) Threedimensional printing of models for preoperative planning and simulation of transcatheter valve replacement. Ann Thorac Surg 93(2):e31-e33

2. Noecker AM, Chen JF, Zhou Q, White RD, Kopcak MW, Arruda MJ (2006) Development of patient-specific three-dimensional pediatric cardiac models. ASAIO J 52:349-353
3. Shiraishi I, Yamagishi M, Hamaoka K, Fukuzawa M, Yagihara T (2010) Simulative operation on congenital heart disease using rubber-like urethane stereolithographic biomodels based on 3D datasets of multislice computed tomography. Eur J Cardiothorac Surg 37:302-306

4. Kiraly L, Tofeig M, Jha NK, Talo H (2016) Three-dimensional printed prototypes refine the anatomy of post-modified Norwood1 complex aortic arch obstruction and allow presurgical simulation of the repair. Interact Cardiovasc Thorac Surg. 22:238-240

5. Schmauss D, Juchem G, Weber S, Gerber N, Hagl C, Sodian R (2014) Three-dimensional printing for perioperative planning of complex aortic arch surgery. Ann Thorac Surg 97:2160-2163

6. Dickinson KJ, Matsumoto J, Cassivi SD, Reinersman JM, Fletcher JG, Morris J et al (2015) Individualizing management of complex esophageal pathology using three-dimensional printed models. Ann Thorac Surg 100:692-697

7. Feins RH, Burkhart HM, Coore DN, Conte JV, Fann JI, Hicks GL (2015) Cardiac surgery simulation curriculum: simulation-based training in surgical skills and decision making. Thoracic Surgery Directors Association, Chapel Hill

8. Hermsen JL, Burke TM, Seslar SP, Owens DS, Ripley BA, Mokadam NA, Verrier ED (2017) Scan, plan, print, practice, perform: development and use of a patient-specific 3-dimensional printed model in cardiac surgery. $\mathrm{J}$ Thorac Cardiovasc Surg 153:132-140

9. Olivieri LJ, Su L, Hynes CF, Krieger A, Alfares FA, Ramakrishnan K, Zurakowski D, Marshall MB, Kim PC, Jonas RA, Nath DS (2016) "Just-In-Time" Simulation Training Using 3-D Printed Cardiac Models After Congenital Cardiac Surgery. World J Pediatr Congenit Heart Surg 7(2):164-168

10. Costello JP, Olivieri LJ, Su L, Krieger A, Alfares FA, Thabit O, Marshall MB, Yoo SJ, Kim PC, Jonas RA, Nath DS (2015) Incorporating three-dimensional printing into a simulation-based congenital heart disease and critical care training curriculum for resident physicians. Congenit Heart Dis 10(2):185-190 